\title{
On the taxonomy of some neotropical species of jumping spiders described by Caporiacco (Araneae, Salticidae)
}

\author{
Gustavo R.S. Ruiz ${ }^{1}$; Antonio D. Brescovit ${ }^{1} \&$ Arno A. Lise ${ }^{2}$ \\ ${ }^{1}$ Laboratório de Artrópodes, Instituto Butantan. Avenida Vital Brazil 1500, 05503-900 São Paulo, São Paulo, Brasil. \\ E-mail: gustavoruiz86@hotmail.com; adbresc@terra.com.br \\ 2 Pontifícia Universidade Católica. Avenida Ipiranga 6681, Prédio 12 C, Sala 244, Caixa Postal 1429, 90619-900 Porto \\ Alegre, Rio Grande do Sul, Brasil. E-mail: lisearno@pucrs.br
}

\begin{abstract}
Pseudattulus incertus Caporiacco, 1955 is synonymized with P. kratochvili Caporiacco, 1947 and the male of this species is redescribed. The genus Uspachus Galiano, 1995 is synonymized with Romitia Caporiacco, 1947 and the male of its type species, $R$. nigra Caporiacco, 1947, is redescribed. The following new combinations are established for the species formerly included in Uspachus: Romitia albipalpis (Taczanowski, 1878), Romitia andina (Taczanowski, 1878), Romitia bahiensis (Galiano, 1995), Romitia colombiana (Galiano, 1995), Romitia juquiaensis (Galiano, 1995), Romitia ministerialis (C.L. Koch, 1846), Romitia misionensis (Galiano, 1995) and Romitia patellaris (Galiano, 1995). The genus Pseudamphidraus Caporiacco, 1947 is synonymized with Noegus Simon, 1900 by transfer of its type species, Noegus niger (Caporiacco, 1947) comb. nov., whose male is herein redescribed. The species Pseudamphidraus variegatus Caporiacco, 1947 and Marma trifidocarinata Caporiacco, 1947 are synonymized with Marma nigritarsis (Simon, 1900).

KEY WORDS. Marma; Noegus; Pseudamphidraus; Pseudattulus; Romitia; Uspachus.
\end{abstract}

RESUMO. Sobre a taxonomia de algumas espécies neotropicais de aranhas papa-moscas descritas por Caporiacco (Araneae, Salticidae). Pseudattulus incertus Caporiacco, 1955 é sinonimizada com P. kratochvili Caporiacco, 1947 e o macho desta espécie é redescrito. O gênero Uspachus Galiano, 1995 é sinonimizado com Romitia Caporiacco, 1947 e o macho de sua espécie-tipo, R. nigra Caporiacco, 1947, é redescrito. As seguintes combinações novas são estabelecidas para as espécies até o momento incluídas em Uspachus: Romitia albipalpis (Taczanowski, 1878), Romitia andina (Taczanowski, 1878), Romitia bahiensis (Galiano, 1995), Romitia colombiana (Galiano, 1995), Romitia juquiaensis (Galiano, 1995), Romitia ministerialis (C.L. Koch, 1846), Romitia misionensis (Galiano, 1995) e Romitia patellaris (Galiano, 1995). O gênero Pseudamphidraus Caporiacco, 1947 é sinonimizado com Noegus Simon, 1900 pela transferência de sua espécie-tipo, Noegus niger (Caporiacco, 1947) comb. nov., cujo macho é redescrito. As espécies Pseudamphidraus variegatus Caporiacco, 1947 e Marma trifidocarinata Caporiacco, 1947 são sinonimizadas com Marma nigritarsis (Simon, 1900).

PALAVRAS-CHAVE. Marma; Noegus; Pseudamphidraus; Pseudattulus; Romitia; Uspachus.

Lodovico di Caporiacco described over 60 species and proposed 10 genera of jumping spiders from the Neotropical Region (CAPORIACCO 1938, 1947, 1948, 1954, 1955), contributing substantially to the knowledge on neotropical diversity of salticids. His descriptions, however, were very poor and no modern taxonomist has had until now the opportunity to examine type specimens of many of his species. Therefore most of Caporiacco's taxa have remained unrecognizable.

Ruiz \& BRESCOVIT (2005) examined the available type specimens of the Venezuelan species described by CAPORIACCO (1955) and established several taxonomic changes. The present study is a second attempt to clarify the identity of some of Caporiacco's neotropical species. Herein, five new synonymies and nine new combinations in the family Salticidae are reported.

\section{MATERIAL AND METHODS}

The material examined was kindly sent to us by the curator of the Museo Zoologico de "La Specola" (MZLS), Firenze, Dr. Sarah Whitman. The measurements are given in millimeters. The abbreviations used throughout the text are: RTA: retrolateral tibial apophysis, d: dorsal, p: prolateral, r: retrolateral, v: ventral, pr: proximal, di: distal.

\section{Sitticinae Simon, 1901}

\section{Pseudattulus Caporiacco, 1947}

Pseudattulus Caporiacco, 1947: 34 (Type species by original designation: Pseudattulus kratochvili Caporiacco, 1947); Caporiacco, 1948: 728; Platnick, 2007.

Revista Brasileira de Zoologia 24 (2): 376-381, junho 2007 


\section{Pseudattulus kratochvili Caporiacco, 1947}

\section{Figs $1-5$}

Pseudattulus kratochvili Caporiacco, 1947: 34 (Male lectotype and two male and one female paralectotypes from Espandaur, Cumins Lodge, Guyana, 9.VIII.1936, Romiti leg., designated by Galiano, deposited in MZLS 598, examined); Caporiacco, 1948: 729, figs 162-163; Berdondini \& Whitman, 2002: 146; Platnick, 2007.

Pseudattulus incertus Caporiacco, 1955: 446, fig. 82 (Female holotype from Maiquetía, Distrito Federal, Venezuela, IX.1948, Marcuzzi leg., deposited in Museo de la Universidad Central de Venezuela, Caracas, number XII-929, examined); Ruiz \& Brescovit, 2005: 756; Platnick, 2007 - Syn. nov.

Sitticus cabellensis Prószynski, 1971: 198, figs 31-39 (Female holotype from Puerto Cabello, Carabobo, Venezuela, E. Simon leg., deposited in Muséum National d'Histoire Naturelle, Paris, not examined; synonymized with Pseudattulus incertus Caporiacco by Ruiz \& BRESCOVIT 2005).

Redescription. Male (lectotype). Total length: 2.85 (Fig. 1). Carapace brown, 1.98 long, 1.36 wide, 0.88 high (Fig. 2), with a wide stripe of white hairs on borders. Ocular quadrangle 0.88 long. Anterior eye row 1.21 wide, posterior 1.28 wide. Chelicera brown, with no tooth on retromargin and three on promargin (Fig. 3). Endite, labium and sternum brown. Palp brown, with a short tibia and a very elongated RTA, embolus short and curved (Figs 4-5); many white hairs dorsally on all the palpal articles. Legs $1>4>2>3$, brown. Length of femur I 1.20, II 0.84, III 0.86, IV 1.34; patella + tibia I 1.70, II 1.12, III 0.92, IV 1.38; metatarsus + tarsus I 1.20, II 0.86, III 0.86, IV 1.14. Spination: femur I d1-1-1, p2di; II = III = IV d1-1-1, p2di, r2di; patella I = II p1; III = IV p1, r1; tibia I v1r-0-1r-1p-2 or v20-1r-1p-2; II v1r-1r-2, p1-0-1; III v1p-2di or v2di, p1-1-1, r1-11; IV d1rpr-1, v2di, p1-1-1, r1-1-1; metatarsus I = II v2-2; III v2di, p2-2, r1-1-2; IV v1p-1p-2 or v1p-2, p1-1-2, r1-1-2. Abdomen black, with a dorsal scutum. Spinnerets black.

Female. See Prószynski (1971): 199 (under Sitticus cabellensis). Distribution. Known from Venezuela and Guyana.

Other material examined. None.

Note. The female holotype and the male allotype of Sitticus cabellensis Prószynski have been mismatched. The female bears the specific name and is identical to that of Pseudattulus kratochvili, hence the synonymy. The male described by Prószynski probably belongs to a new genus.

\section{“Freya-group" Romitia Caporiacco, 1947}

Romitia Caporiacco, 1947: 32 (Type species by original designation: Romitia nigra Caporiacco, 1947); Caporiacco, 1948: 716; Platnick, 2007.

Uspachus Galiano, 1995: 122 (Type species by original designation: Uspachus misionensis Galiano, 1995); Platnick, 2007 Syn. nov.
Remarks. According to GaLIANO (1995), the genus Uspachus may be related to Phiale C.L. Koch, 1846 and Pachomius Peckham $\&$ Peckham, 1896, but differs from them mainly by presenting a quadrangular membranous lamella - or simply a distal lamella near the embolus, on the embolic division of the tegulum (GALIANO 1995: 122, figs 1-2). This structure is also present in the type species of Romitia, $R$. nigra, which supports the synonymy. It is possible that the distal lamella in Romitia is homologous to the "denticles" or the "tegular projections" present in some species of Pachomius (see Galiano 1994, figs 8-10, 15-17), and that some features delimiting Romitia, like the transverse epigynal furrow, although apomorphic for the lineage, can turn Pachomius into a paraphyletic cluster, suggesting that both genera should be synonymized in the future.

Note. The new combinations in Romitia are established based on Galiano's illustrations (GaLiano 1995). No type has been examined. For a complete list of synonyms and citations of these species, see Platnick (2007).

\section{Romitia nigra Caporiacco, 1947}

Figs 6-9

Romitia nigra Caporiacco, 1947: 32 (Male holotype from Garroway Landing, Potaro, Guyana, 30.III.1936, Romiti leg., deposited in MZLS 599, examined); Caporiacco, 1948: 716, fig. 147; Berdondini \& Whitman, 2002: 147; Platnick, 2007.

Diagnosis. Romitia nigra Caporiacco differs from all the remaining species of the genus by having a distal lamella as long as the embolus that crosses over the embolus of the male palp in ventral view (Fig. 6).

Redescription. Male (holotype). Total length: 4.50. Carapace dark brown, 2.40 long, 1.60 wide, 1.00 high, with a wide stripe of white hairs on borders. Ocular quadrangle 1.20 long. Anterior eye row 1.40 wide, posterior 1.35 wide. Chelicera dark brown, with one tooth on retromargin and two on promargin. Endite dark brown, like that of Romitia misionensis (Galiano, 1995; see below) (see Galiano 1995, fig. 7). Labium and sternum dark brown. Palp dark brown (Figs 6-9), femur dorsally dilated with a granulated field ventrally on the distal extremity as that of Romitia misionensis (Galiano, 1995) (see GaLIANO 1995, fig. 6); RTA short, embolus dorsal, covered by a ventral distal lamella. Legs $4>1>3>2$, dark brown. Length of femur I 1.37, II 1.10, III 1.37, IV 1.50; patella + tibia I 1.75, II 1.30, III 1.35, IV 1.60; metatarsus + tarsus I 1.30, II 1.05, III 1.45, IV 1.67. Spination: femur I d1-1-1, p2di; II d1-1-1, p2di, r1di; III = IV d1-1-1, p2di, r2di; patella I = II p1; III = IV p1, r1; tibia I v22-2, p1-0-1; II v1r-2-2, p1-0-1, r1pr or r0; III = IV d1pr, v1p-0-2, p1-1-1, r1-1-1; metatarsus I = II v2-2; III = IV d1, v2-2, p1-0-2, r1-0-2. Abdomen dark brown, with a median dorsal tuft of white hairs close to the spinnerets.

Female. Unknown.

Distribution. Known only from Guyana.

Other material examined. None.

Revista Brasileira de Zoologia 24 (2): 376-381, junho 2007 


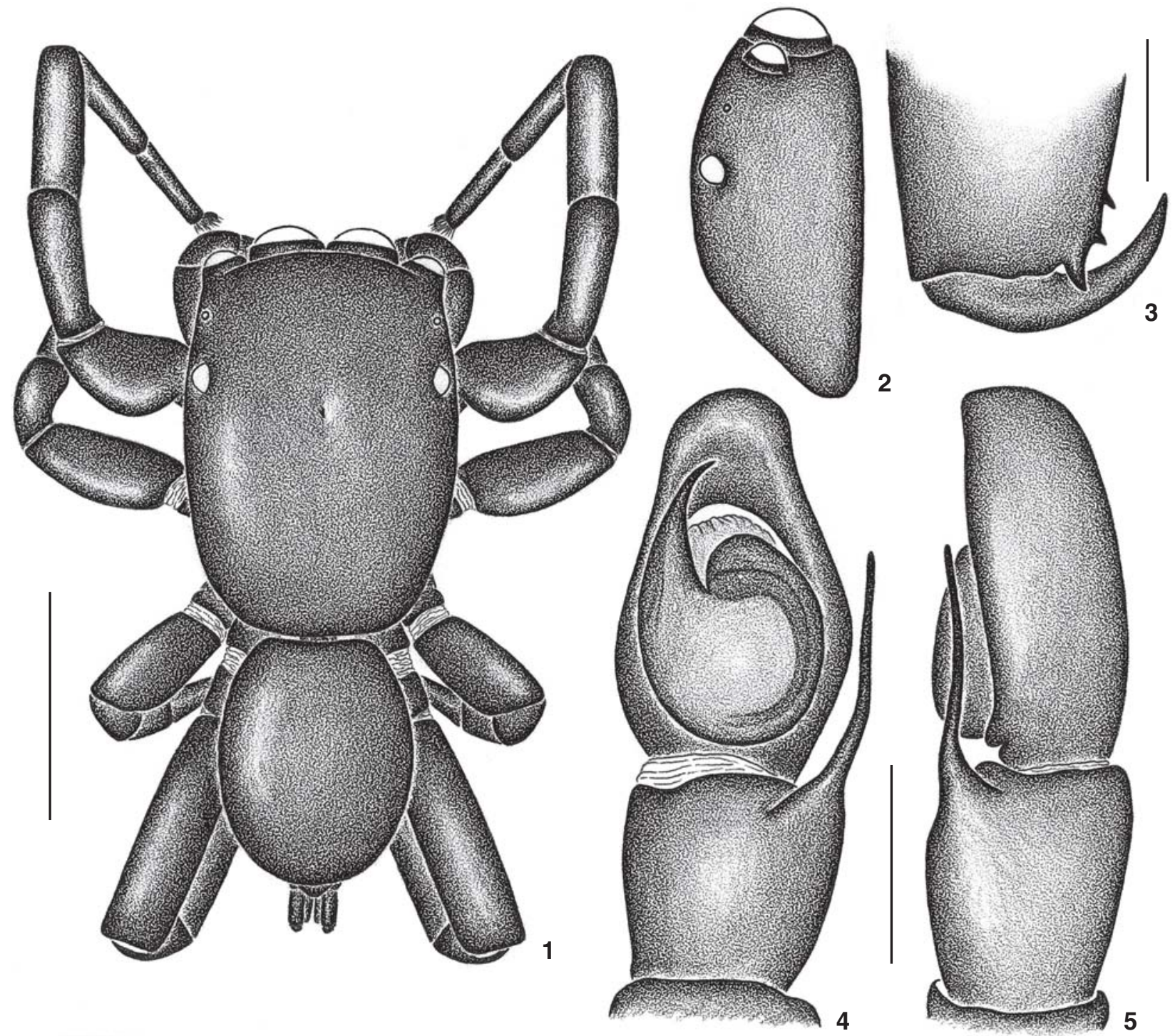

Figures 1-5 Pseudattulus kratochvili: (1) male, dorsal view; (2) male carapace, lateral view; (3) male chelicera, anterior view; (4) male palp, ventral view; (5) retrolateral view. Scale lines: (1-2) $1 \mathrm{~mm}$; (3-5) $0.25 \mathrm{~mm}$.

Romitia albipalpis (Taczanowski, 1878) comb. nov. Euophrys albipalpis Taczanowski, 1878: 291.

Uspachus albipalpis: Galiano, 1995: 135, figs 17-18, 22, 45-47, 50; Platnick, 2007.

Romitia andina (Taczanowski, 1878) comb. nov. Euophrys andina Taczanowski, 1878: 297.

Uspachus andinus: Galiano, 1995: 139, figs 53-54; Platnick, 2007.

\section{Romitia bahiensis (Galiano, 1995) comb. nov.}

Uspachus bahiensis Galiano, 1995: 138, figs 19-20, 23, 30, 3536, 49; Platnick, 2007.
Romitia colombiana (Galiano, 1995) comb. nov.

Uspachus colombianus Galiano, 1995: 137, figs 11, 39, 41-42; Platnick, 2007.

Romitia juquiaensis (Galiano, 1995) comb. nov.

Uspachus juquiaensis Galiano, 1995: 138, figs 15-16, 24, 40, $43-$ 44; Platnick, 2007.

Romitia ministerialis (C.L. Koch, 1846) comb. nov. Phiale ministerialis C.L. Koch, 1846: 199, figs 1247.

Uspachus ministerialis: Galiano, 1995: 134, figs 12-14, 33-34; Platnick, 2007.

Revista Brasileira de Zoologia 24 (2): 376-381, junho 2007 

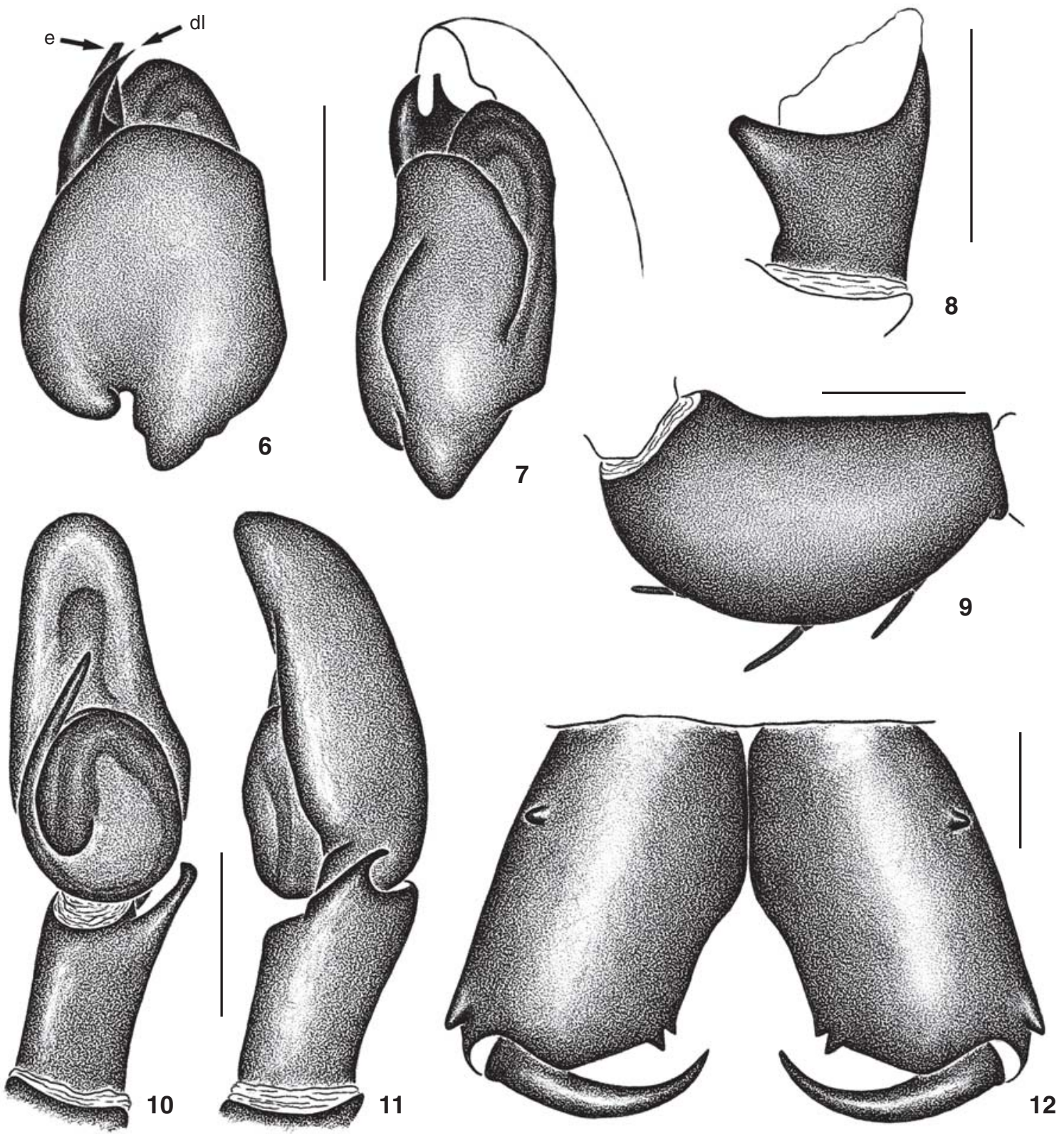

Figures 6-12. (6-9) Romitia nigra: (6) male palp, ventral view; (7) ventro-retrolateral view; (8) palpal tibia, retrolateral view; (9) palpal femur, lateral view; (10-12) Noegus niger: (10) male palp, ventral view; (11) retrolateral view; (12) male chelicerae, anterior view. Abbreviations: (e) embolus; (dl) distal lamella. Scale lines: $0.25 \mathrm{~mm}$.

Romitia misionensis (Galiano, 1995) comb. nov.

Uspachus misionensis Galiano, 1995: 129, figs 1-10, 31-32, 3738; Platnick, 2007.

\section{Romitia patellaris (Galiano, 1995) comb. nov.}

Uspachus patellaris Galiano, 1995: 140, figs 21, 25-29, 48, 5152; Platnick, 2007.

\section{Amycinae Simon, 1901 \\ Noegus Simon, 1900}

Noegus Simon, 1900: 50 (Type species by original designation: Noegus vulpio Simon, 1900); Platnick, 2007.

Beccaria Caporiacco, 1947: 30 (Type species by original designation: Beccaria franganilloi Caporiacco, 1947); Caporiacco, 1948: 703; Galiano, 1982: 423 (syn.).

Revista Brasileira de Zoologia 24 (2): 376-381, junho 2007 
Pseudamphidraus Caporiacco, 1947: 30 (Type species by original designation: Pseudamphidraus niger Caporiacco, 1947); Caporiacco, 1948: 700; Platnick, 2007 - Syn. nov.

Remarks. The type species of Pseudamphidraus Caporiacco, $P$. niger Caporiacco, presents the same dentition as the species included in Noegus Simon, with several retromarginal teeth and a pair on the promargin, the synapomorphy of the genus (Galiano, 1968: 345).

\section{Noegus niger (Caporiacco, 1947) comb. nov.} Figs 10-12

Pseudamphidraus niger Caporiacco, 1947: 30 (Male holotype from Campo V, Demerara, Guyana, XI.1931, Beccari leg., deposited in MZLS 596, examined); Caporiacco, 1948: 700, fig. 124; Berdondini \& Whitman, 2002: 147; Platnick, 2007.

Diagnosis. Noegus niger (Caporiacco) resembles N. actinosus Simon, 1900 by having the tip of the simple RTA projecting dorsally (see GaLiano 1963, tab. 29, fig. 1), but can be distinguished from this species by having two pairs of external, very small mastidia on the chelicerae (Fig. 12).

Redescription. Male (holotype). Total length: 4.50. Carapace dark brown, 2.20 long, 1.75 wide, 1.50 high. Ocular quadrangle 1.50 long. Anterior eye row 1.70 wide, posterior 1.55 wide. Chelicera dark brown, with five teeth on retromargin and two on promargin (Fig. 12). Endite, labium and sternum dark brown. Palp dark brown, tegulum globose, embolus long and narrow (Figs 10-11). Legs $1>3=4>2$, dark brown; metatarsi and tarsi light brown. Length of femur I 1.85, II 1.55, III 1.75, IV 1.55; patella + tibia I 2.35, II 1.75, III 1.75, IV 1.70; metatarsus + tarsus I 1.65, II 1.40, III 1.55, IV 1.80. Spination: femur I = II = III d1-1-1, p2di, r1di; IV d1-1-1, p1di, r1di; patella I 0; II p1; III = IV p1, r1; tibia I v2-2-2, p1-0-1; II v1r-2-2, p1-1-1; III v1p-0-1p, p1-0-1, r1-1-1; IV v1p-0-1r, p1-0-1, r1-1-1; metatarsus I = II v2-2; III v2-2, p1-0-2, r1-0-2; IV v1p-2, p1-0-2, r1-1-2. Abdomen dark brown, dorsally variegated with dark brown and yellow.

Female. Unknown.

Distribution. Known only from Guyana.

Other material examined. None.

\section{Amycoida incertae sedis \\ Marma Simon, 1902}

Marma Simon, 1902: 376 (Type species by original designation: Marma baeri Simon, 1902); Platnick, 2007.

Thysema Mello-Leitão, 1944: 390 [Type species by original designation: Thysema dorae Mello-Leitão, 1944 = Marma nigritarsis (Simon, 1900)]; Galiano, 1962: 36 (syn.).

Paralophostica Soares \& Camargo, 1948: 396 [Type species by original designation: Paralophostica centralis Soares \& Camargo, 1948 = Marma nigritarsis (Simon, 1900)]; Galiano, 1962: 36 (syn.).

Revista Brasileira de Zoologia 24 (2): 376-381, junho 2007

\section{Marma nigritarsis (Simon, 1900)}

Amphidraus nigritarsis Simon, 1900: 60; Simon, 1901: 419, fig. 477.

Marma nigritarsis: Galiano, 1962: 40, pl. I, figs 6-9, pl. II, figs 45; Galiano, 1963: 397, pl. XXVIII, figs 1-2; Galiano, 1981: 12; Platnick, 2007.

Pseudamphidraus variegatus Caporiacco, 1947: 30 (Male holotype from Two Mouths, Esequibo, Guyana, 14.VII.1936, Romiti leg., deposited in MZLS 597, examined); Caporiacco, 1948: 700, fig. 125; Berdondini \& Whitman, 2002: 149; Platnick, 2007 - Syn. nov.

Marma trifidocarinata Caporiacco, 1947: 34 (Female holotype from Alto Demerara, Guyana, XII.1931, Beccari leg., deposited in MZLS 549, examined); Caporiacco, 1948: 731, fig. 165; Berdondini \& Whitman, 2002: 149; Platnick, 2007 - Syn. nov.

Note. For a complete list of synonyms and citations of Marma nigritarsis (Simon), see PlatNICK (2007).

Remarks. The synonymies were detected through comparisons among Caporiacco's types, specimens deposited in the collection of Instituto Butantan and the excellent illustrations of Marma nigritarsis by GaLIANO (1962, tab. 1, figs 6-9; tab. 2, figs 4-5).

\section{ACKNOWLEDGMENTS}

We wish to thank the Coordenação de Aperfeiçoamento de Pessoal de Nível Superior (CAPES - GRSR) and the Conselho Nacional de Desenvolvimento Científico e Tecnológico (CNPq ADB) for financial support. We also thank the curator of the Museo Zoologico de "La Specola", Sarah Whitman, for sending the type specimens, and G.B. Edwards for the comments on the manuscript. This study was developed in the Programa de Pós-Graduação do Instituto de Biociências da Universidade de São Paulo.

\section{REFERENCES}

Berdondini, I. \& S. Whitman. 2002. Cataloghi del Museo di Storia Naturale dell'Università di Firenze - sezione di zoologia «La Specola». XVI. Arachnida, Araneae: Tipi. Atti de la Società Toscana di Scienze Naturali 109: 119-156.

Caporiacco, L. di. 1938. Aracnidi del Messico, di Guatemala e Honduras britannico. Atti della Società Italiana de Scienze Naturali 77: 251-282.

Caporiacco, L. di. 1947. Diagnosi preliminari de specie nuove di aracnidi della Guiana Brittanica raccolte dai professori Beccari e Romiti. Monitore Zoologico Italiano 56: 20-34.

Caporiacco, L. di. 1948. Arachnida of British Guiana collected in 1931 and 1936 by Professors Beccari and Romiti. Proceedings of the Zoological Society of London 118: 607-747.

Caporiacco, L. di. 1954. Araignées de la Guyane Française du Muséum d'Histoire Naturelle de Paris. Commentaitiones Pontificiae Academiae Scientiarum 16: 45-193.

CAPORIACCO, L. di. 1955. Estudios sobre los arácnidos de Venezuela. 2a parte: Araneae. Acta Bioliologica Venezuelica 1: 265-448. Crane, J. 1949. Comparative biology of salticid spiders at Ran- 
cho Grande, Venezuela III, IV. Zoologica 34: 31-52, 159 214.

Galiano, M.E. 1962. Los géneros Amphidraus Simon y Marma Simon (Araneae, Salticidae). Acta Zoologica Lilloana 18: $31-44$

Galiano, M.E. 1963. Las especies americanas de arañas de la familia Salticidae descriptas por Eugène Simon: Redescripciones basadas en los ejemplares típicos. Physis (C) 23: 273-470.

Galiano, M.E. 1968. Revisión de los géneros Acragas, Amycus, Encolpius, Hypaeus, Mago y Noegus (Salticidae, Araneae). Revista del Museo Argentino de Ciencias Naturales Bernardino Rivadavia (Entomología) 2: 267-360.

Galiano, M.E. 1981. Catálogo de los especímenes típicos de Salticidae (Araneae) descriptos por Cândido F. de Mello-Leitão. Segunda parte. Physis (C) 39: 11-17.

Galiano, M.E. 1982. New combinations and synonymies in Salticidae (Araneae). Bulletin of the British Arachnological Society 5: 423-424.

Galiano, M.E. 1994. Revision of the genus Pachomius (Araneae, Salticidae). Bulletin of the British Arachnological Society 9: 214-220.

Galiano, M.E. 1995. Descripción de Uspachus, nuevo género (Araneae, Salticidae). Physis (C) 50: 121-142.

Mello-Leitão, C.F. de. 1941. Las arañas de Córdoba, La Rioja, Catamarca, Tucumán, Salta y Jujuy colectadas por los Profesores Birabén. Revista del Museo de La Plata (Zoología) 2: 99-198.
Mello-Leitão, C.F. de. 1944. Arañas de la provincia de Buenos Aires. Revista del Museo de La Plata (N.S., Zoología) 3: 311393.

Platnick, N.I. 2007. The world spider catalog. American Museum of Natural History, Version 7.5. Avalable on line at: http://research.amnh.org/entomology/spiders/catalog/ index.html [Accessed in 03.V.2007]

PrószYNSKI, J. 1971. Revision of the spider genus Sitticus Simon, 1901 (Aranei, Salticidae). II. Sitticus saxicola (C.L. Koch, 1848) and related forms. Annales Zoologici 28: 183-204.

Ruiz, G.R.S. \& A.D. Brescovit. 2005. Notes on the Venezuelan jumping spiders described by Caporiacco (Araneae, Salticidae). Revista Brasileira de Zoologia 22: 753-760.

Simon, E. 1900. Études arachnologiques. 30e Mémoire. XLVII. Descriptions d'espèces nouvelles de la famille des Attidae. Annales de la Société Entomologique de France 69: 27-61. Simon, E. 1901. Histoire naturelle des araignées. Paris, 2: 381-668. Simon, E. 1902. Description d'arachnides nouveaux de la famille des Salticidae (Attidae) (suite). Annales de la Société Entomologique de Belgique 46: 24-56, 363-406.

Soares, B.A.M. \& H.F. de A. Camargo. 1948. Alguns novos salticidas do Brasil (Araneae, Salticidae). Revista Brasileira de Biologia 8: 421-443.

Taczanowski, L. 1878. Les Aranéides du Pérou. Famille des Attides. Buletin de la Société Impériale des Naturalistes de Moscou 53: 278-374.

Received in 31.X.2006; accepted in 03.V.2007.

Revista Brasileira de Zoologia 24 (2): 376-381, junho 2007 